\title{
Dynamic tensile test of single PET textile cables
}

\author{
P. Guégan ${ }^{1}$, R. Othman ${ }^{1}$, F. Pasco ${ }^{1}$, and R. Bruant ${ }^{2}$ \\ ${ }^{1}$ GeM Institute, École Centrale Nantes, Université LUNAM, 1 rue de la Noë, BP. 92101, 44321 Nantes Cedex \\ 3, France \\ ${ }^{2}$ MICHELIN, Ladoux, 63040 Clermont Ferrand Cedex 9, France
}

\begin{abstract}
The tyres conception involves for certain applications, the use of textile cables as reinforcement. During its use, the tyre undergoes temperatures variations and dynamic loading rates. The consideration of these conditions during the numeric simulations requires the knowledge of the sensitivity of the mechanical behaviour to loading rate and temperature. In this paper, we developed an experimental methodology for testing textile cable up to high strain rate. The main difficulty of testing cables is the optimization of cable fixing on the machine. For that purpose, we adapted the solution of fixing by progressive binding already used in quasi-static, while taking into account constraints inherent to high strain tests. Firstly, the mass of grips was decreased in order to get force signal less sensitive to grips inertia. The method was developed on a high speed hydraulic machine equipped with a thermal enclosure. The investigated temperatures and strain rates range from room temperature to $373^{\circ} \mathrm{K}\left(100^{\circ} \mathrm{C}\right)$ and from 0,01 to $100 / \mathrm{s}$, respectively. In addition, the hydraulic machine was equipped with a high speed video camera. The obtained images were analysed by a tracking technique to measure the average strain in the cable (from 50 to $20000 \mathrm{f} / \mathrm{s}$ ).
\end{abstract}

\section{Introduction}

Fibre-reinforced rubber composites are widely used nowadays because of their processing advantage and good technical properties such as strength and stiffness [1]. One of their multiple applications is tyre industry. For this industry and others, the knowledge of the mechanical behaviour of fibres and its sensitivity to temperature and strain rate is a main concern.

In this manuscript, we are interested in measuring the mechanical behaviour of PET fibres for temperatures at and higher than room temperature. We are also concerned with the assessing the fibres mechanical properties at quasi-static and intermediate strain rates. The obtained data will be used to develop numerical models of tyres and then to optimize their dimensions.

However, testing single fibres is much difficult. The difficulty is much harder to solve when testing at intermediate or high strain rates. Consequently, few papers addressed this point in literature [2-6]. The main difficulties are (i) to attach the fibre to the mechanical testing machine and (ii) to measure low level forces.

In order to sidestep these problems, one can study either fabric armours [7] or fibre bundles [8-11]. However, the response of fabrics and bundles is always structural and can not account for the response of a single fibre or cable.

The aim of the present work is to measure the forceelongation curves, up to break, of textile cables at dynamic loading rates. This study will mainly include:

- Realization of a specific grip to fix cables on the testing machine; the grip geometry should guide breaking to happen in the gauge zone and not in the fixation parts.

- Checking of force transducer sensitivity regarding the forces applied to the fibre.

- Validation of the imposed tensile velocities regarding the desired strain rates.
The present developed methodology will then be applied to measure temperature and strain rate sensitivity of the PET fibre mechanical properties.

\section{Method}

\subsection{Material}

In this study, we will deal with PET textile cables containing several stalks. Each of the stalks is constituted by a large number of thread. In quasi-static, the strength is approximately $200 \mathrm{~N}$, and the order of the ultimate strain is $15 \%$.

\subsection{Scope statement}

The objective of the experimental work is to realize mechanical tests on a single PET cable at different strain rates, namely, 0.01, 0.1,1, 10 and $100 \mathrm{~s}^{-1}$. Moreover, the temperature sensitivity should be investigated. Precisely, three temperatures will be considered: room temperature (between 20 and $25^{\circ} \mathrm{C}$ ), 50 and $100^{\circ} \mathrm{C}$. On the other hand, the influence of hygrometry will be ignored. In order to check experimental results scatter, three tests will be carried out for identical experimental parameters.

The single cable will be fixed on the testing machine by progressive binding and will be submitted to a pre-tensile force around $1 \mathrm{~N}$.

\subsection{Grips}

The cable is fixed on the machine by a progressive binding on the grips. The geometry of grips used for quasi-static experiments is shown in figure 1. Two grips are used. The upper one is attached to the force sensor and the lower 


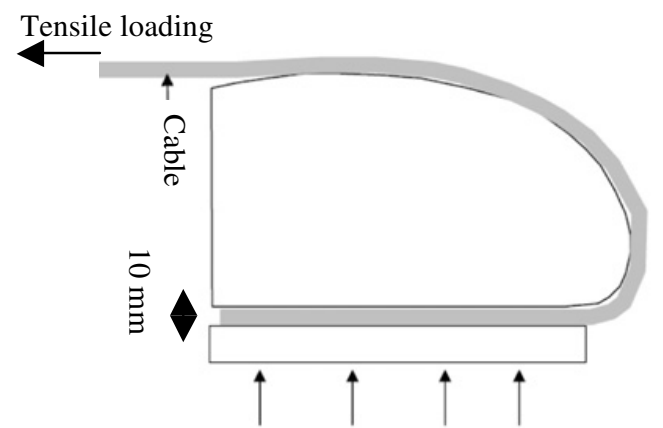

Fig. 1. Geometry of the grips used for the quasi-statics experiments. $($ dim. $100 \times 50 \mathrm{~mm})$.

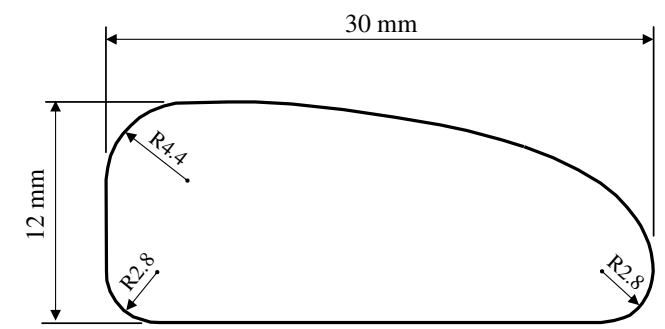

Fig. 2. Geometry of the shape conceived for the study.

one is fixed to the moving jack. For dynamic strain rate experiments, smaller grips were considered (figure 2) as the inertia of quasi-static grips too important for dynamic tests.

Actually, the single fibres are fixed by the friction efforts resulting from the contact between the cable and the grips. This property allows limiting stress concentrations in the cable. Therefore, the cable breaking will be in the gauge zone and not in the fixing parts.

\subsection{Testing machine}

The tests are realized on a high velocity hydraulic machine MTS 810. The tensile force is measured with a piezoelectric transducer of a maximum capacity of $\pm 2,5 \mathrm{kN}$. The sensor is attached to the upper grip.

The lower grip is attached to a piston which allows a free movement of the hydraulic jack before achieving the desired constant velocity. The piston and lower grip are dimensioned so that pre-tensile force imposed to the fibre is near $1 \mathrm{~N}$. The fibre once attached to the grips is shown in figure 3.

\subsection{Strain measurement}

The deformation of the fibre is obtained by tracking analysis of images. The experimental set-up is equipped with a high speed video camera: PHOTRON APX (the maximum resolution $1024 \times 128$ pix $^{2}$ and the acquiring frequency is between 60 and 15000 f/s). Two felt-tip marks are realised on the cable (figure 4) in X1 and X2. The points so realized allow tracking the movement of theses two points. To do this, the obtained images are analysed by the software

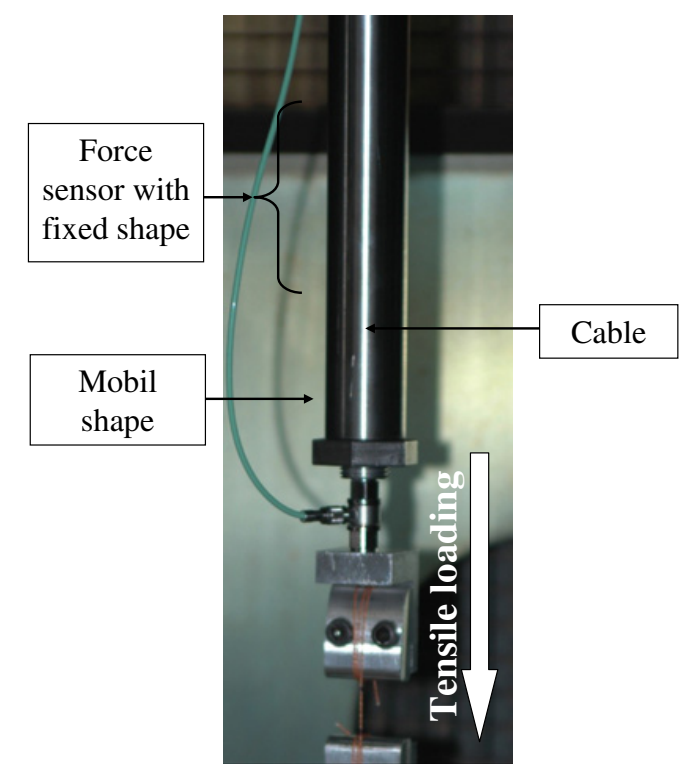

Fig. 3. Experimental set-up.

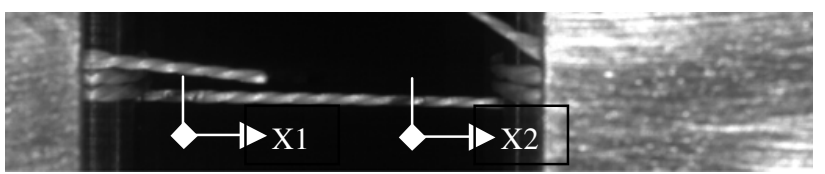

Fig. 4. Example of mark on cable.

TEMA 2D. Hence we can get the displacement of both points $\mathrm{X} 1(\mathrm{t})$ and $\mathrm{X} 2(\mathrm{t})$.

Afterwards, the nominal strain and the nominal strainrate are deduced as follows:

$$
\varepsilon_{(t)}=\frac{\Delta l}{L_{0}}=\frac{(X 2(t)-X 1(t))-(X 2(0)-X 1(0))}{(X 2(0)-X 1(0))}
$$

and

$$
\dot{\varepsilon}=\frac{d}{d t}(\varepsilon)=\frac{d}{d t}\left(\frac{\Delta l}{L_{0}}\right)
$$

respectively.

\subsection{Temperature control}

In order to achieve temperatures different from room temperature a thermal enclosure is used. This enclosure enables controlling temperatures ranging from -150 to $+600^{\circ} \mathrm{C}$. Its door is equipped with a window so that one can use optic extensometer. The cable is thus filmed through this window by the high speed video camera video camera (figure 5).

The uniformity of the temperature in the thermal enclosure is ensured by the circulation of hot/cold air. The force sensor is able to give accurate measurement under environmental temperature lower than $150{ }^{\circ} \mathrm{C}$. Thus, it is placed in the thermal enclosure directly attached to the upper grip. 


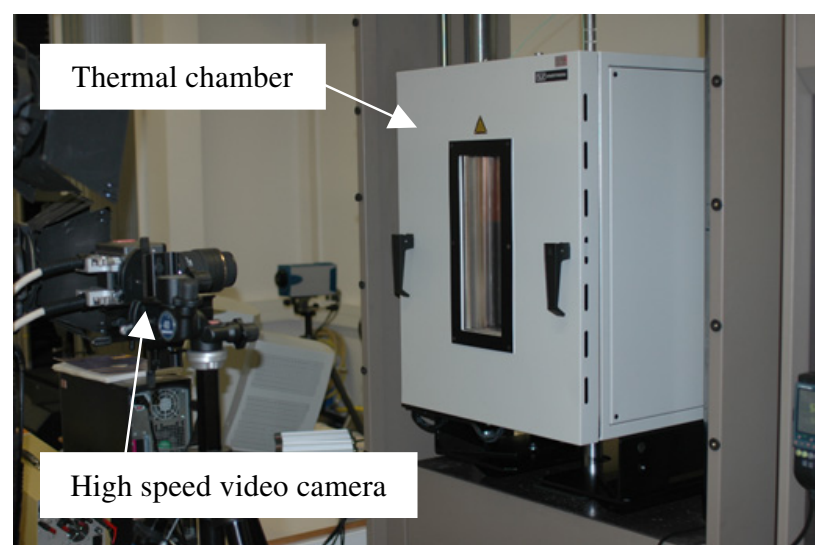

Fig. 5. Thermal enclosure mounted on the MTS hydraulic machine.

Table 1. Experimental program.

\begin{tabular}{|l|c|c|c|c|}
\hline$\dot{\varepsilon}\left(\mathrm{s}^{-1}\right)$ & \multicolumn{3}{|c|}{ Temperature } & Tensile velocity \\
\hline & Ambient & $50{ }^{\circ} \mathrm{C}$ & $10{ }^{\circ} \mathrm{C}$ & applied \\
\hline 0,01 & $\mathrm{X}$ & & & $1 \mathrm{~mm} / \mathrm{s}$ \\
\hline 0,1 & $\mathrm{X}$ & & & $10 \mathrm{~mm} / \mathrm{s}$ \\
\hline 1 & $\mathrm{X}$ & & & $100 \mathrm{~mm} / \mathrm{s}$ \\
\hline 10 & $\mathrm{X}$ & & & $1 \mathrm{~mm} / \mathrm{s}$ \\
\hline 100 & $\mathrm{X}$ & $\mathrm{X}$ & $\mathrm{X}$ & $8 \mathrm{~m} / \mathrm{s}$ \\
\hline
\end{tabular}

\subsection{Test configurations}

Nine experimental configurations (couples of temperature and strain rate) were considered in the experimental program. The details of these configurations are given Table 1 .

The sliding and the contraction of the cable on the grips involve an effective velocity higher than to the desired one.

The procedure of tests at controlled temperature is as follows:

1. Assemble the cable on the two grips.

2. Close the door of the thermal enclosure.

3. Turn on of the thermal enclosure.

4. Reach the desired temperature.

5. Wait for 5 minutes.

6. Realize the mechanical test.

7. Stop the hydraulic jack of the machine and open the chamber.

8. Dismantle of the cable.

\section{Results}

\subsection{Breaking location}

The single fibre fixing is realized by three revolutions of it around the shape. This solution allows stopping the sliding of the cable while avoiding pinching it too much.

For each test we have noted the location where fibre have broken. It comes that breaking happens

- $27 \%$ of tests in the useful part of the fibre (gage part).

- $73 \%$ of tests in the low and high extremities of the gage part.

$-0 \%$ in the grips.

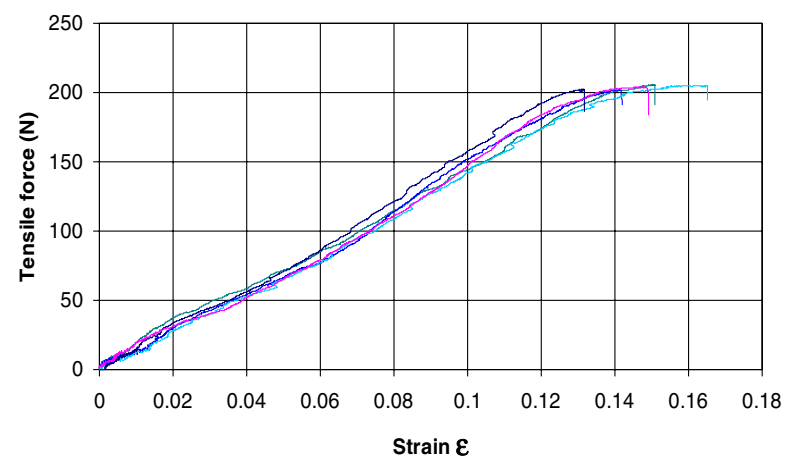

Fig. 6. Example of tensile force-strain curves obtained at a strain rate of $1 \mathrm{~s}^{-1}$ and room temperature.

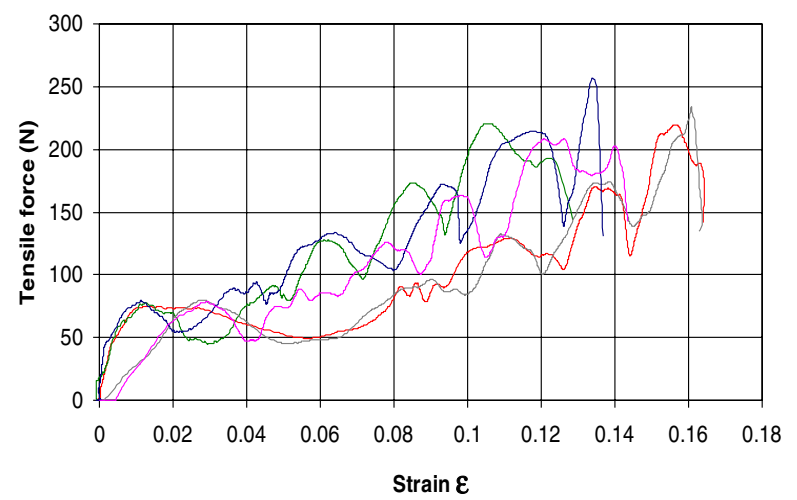

Fig. 7. Example of tensile force-strain curves obtained at a strain rate of $100 \mathrm{~s}^{-1}$ and room temperature.

The new design of grips avoided breaking in these parts. However, for around two-third of the tests the breaking is influenced by the fixing of the fibres. Around onethird of cables have broken in the gauge part. For these tests the grips and fixing worked perfectly. This is a quiet appreciated result for so hard task as fixing a single cable.

\subsection{Experimental curves}

The figure 6 shows an example of experimental curves obtained at $1 \mathrm{~s}^{-1}$ and room temperature. The behaviour is elastic linear followed by a clear rupture.

At a strain rate of $100 \mathrm{~s}^{-1}$, the signals are more altered (figure 7). The observed oscillations may result from wave reverberation in the experimental device and the inertia of the force transducer [12].

\subsection{Ultimate strains}

The average ultimate strains obtained for the different experimental configuration are showed in figure 8 . There is some scatter is the experimental results which is expected in such difficult experiments. This may be yielded by the variation of cable fixing from test to test. The variation of the images quality from test to test can also influence the accuracy of the measured strains. Nevertheless, we can observe some general tendencies. Namely, the average ultimate strain is decreasing with temperature and strain 


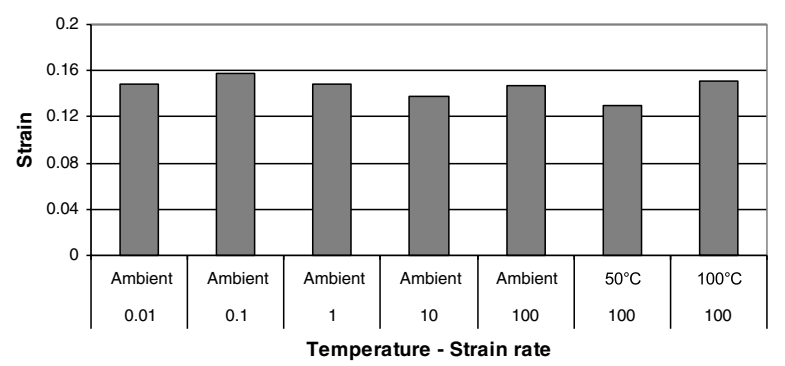

Fig. 8. Average ultimate strains for the different experimental configurations.

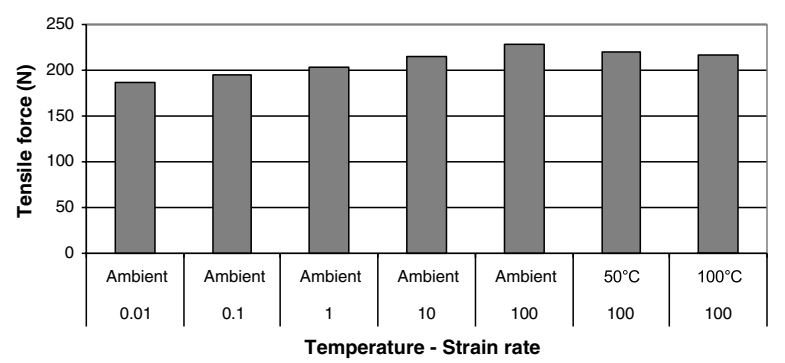

Fig. 9. Average ultimate forces for the different experimental configurations.

rate. The effect of the strain rate is expected. However, the effect of temperature is unusual and should be confirmed by further investigations.

\subsection{Ultimate forces}

The average ultimate forces obtained for the different experimental configurations are depicted in figure 9. The scatter in the experimental results is less than that observed for the average ultimate strains. The accuracy of the force measurements is better than that of strain measurements.

The ultimate force tends to decrease with increasing temperatures. This is expected as temperature has a softening effect. The effect of strain rate is also here unsurprising as the ultimate force is rises up for increasing strain rates. However, the sensitivity of the ultimate force to strain rate is not important.

\section{Conclusion}

Several conclusions can be summed up relative to the work presented in this work.

- The new grips conceived for the dynamic tensile testing of single fibres allow obtaining a satisfactory location of breaking.

- The location of fibre breaking has a slight effect on the measured ultimate forces and strains.

- The scatter in the measured ultimate strains, however acceptable, is higher than the scatter in the measured ultimate forces.

- The average ultimate force tends to decrease with increasing temperature and decreasing strain rates.

- The sensitivity of the ultimate force to strain rate is about $4 \%$ per decade.

- The average ultimate strain is decreasing with increasing temperature and strain rate.

- The effect of temperature on the ultimate strain is quite unusual and should be confirmed or undermined by further investigations.

\section{References}

1. H.A. Youssef, N.A. Shaltot, K.F. El Nemr, A.A. El Miligy, J Polym. Res. 16 (2009).

2. T.A. Godfrey, J. Mater Sci. 38 (2003).

3. B. Farsi Dooraki, J.A., Nemes, M. Bolduc, J. Phys IV 134 (2006).

4. V.B.C. Tan, X.S. Zeng, V.P.W. Shim, Int. J. Impact Eng. 35 (2008).

5. A.C.P. Koh, V.P.W. Shim, V.B.C. Tan, Int. J. Impact Eng. 37 (2010).

6. J. Lim, J.Q. Zheng, K. Masters, W.W. Chen, J. Mater. Sci. 45 (2010)

7. V.P.W. Shim, C.T. Lim, K.J. Foo, Int. J. Impact Eng. 25 (2001)

8. Y. Wang, Y. Xia, Compos. Part A 29 (1998).

9. Y. Wang, Y. Xia, Compos. Part A 30 (1999).

10. W. Huang, Y. Wang, Y. Xia, Polym. 45 (2004).

11. Y. Zhou, Y. Wang, Y. Xia, S. Jeelani, Mater. Lett. 64 (2010).

12. R. Othman, P. Guégan, G. Challita, F. Pasco, D. LeBreton, Int J Impact Eng 36 (2009). 